\title{
97. A Study of the Anomalies in the Vertical Gradient of Gravity with the Aid of the BESSEL FourIER Series
}

\author{
By Chuji Tsubor \\ Geophysical Institute, Faculty of Science, Tokyo University \\ (Comm. by M. Matuyama, M.J.A., June 12, 1954)
}

The vertical gradient of gravity is usually taken to be $3086 \times 10^{-9}$ c.g.s. and the free-air reductions of gravity values are made almost exclusively by use of this numerical value. But to be precise, this quantity is never a constant throughout and varies from place to place in accordance with the variation in the gravity value itself. ${ }^{1}$ That the vertical gradient of gravity does vary so can be seen qualitatively as follows:

Consider two points A and B lying at the same height. Denote the gravity values at the two points by $g_{\mathrm{A}}(h)$ and $g_{\mathrm{B}}(h)$ and assume that $g_{\mathrm{A}}(h)>g_{\mathrm{B}}(h)$. We know that the gravity distribution along a horizontal plane tends to be more and more uniform with height, so that it must be that

$$
g_{\mathrm{A}}(h)-g_{\mathrm{B}}(h)>g_{\mathrm{A}}(h+z)-g_{\mathrm{B}}(h+z) .
$$

From this expression, it naturally follows that

$$
\left\{g_{\mathrm{A}}(h)-g_{\mathrm{A}}(h+z)\right\} / z>\left\{g_{\mathrm{B}}(h)-g_{\mathrm{B}}(h+z)\right\} / z .
$$

The relation (1) states that the decrease of gravity with height is larger at A than at B. It can generally be said that where the gravity anomaly is positive, the decrease of gravity with height is steeper and where the gravity anomaly is negative, the decrease is gentler than it normally is. But in order to see whether or not such anomalies in the vertical gradient play appreciable roles in actual problems, the matter must be investigated quantitatively.

For this purpose, let us study the two-dimensional problem first. If the gravity potential due to a certain anomalous subterranean mass distribution is expressed by

$$
\Delta U(x z)=\frac{1}{2 \pi} \sum_{m} \lambda_{m} B_{m} \begin{aligned}
& \cos 2 \pi \\
& \sin \lambda_{m}
\end{aligned} x \exp \left(-\frac{2 \pi}{\lambda_{m}} z\right),
$$

then, the gravity anomaly along $z=0$ due to that mass distribution is given by

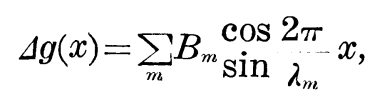

1) C. Tsubor: Journ. Phys. Earth, 1, 97 (1952). 
and the anomaly in the vertical gradient of gravity by

$$
\Delta(\partial g / \partial z)(x)=-2 \pi \sum_{m} \frac{B_{m} \cos 2 \pi}{\lambda_{m}} \sin \lambda_{m} x .
$$

Here $\lambda_{m}$ is the wave length of the $m$-th harmonics when the horizontal distribution of the quantities concerned is expressed in the Fourien series with respect to $x$. From the above expressions (3) and (4), each component of $\Delta(\partial g / \partial z)$ is seen to be negative $2 \pi / \lambda_{m}$ times as large as the corresponding component of $\Delta g$. The shorter wave length variation in $\Delta g$ affects $\Delta(\partial g / \partial z)$ more than the longer wave length variation does, provided $B_{m}$ 's for both cases are the same. The negative sign comes from the circumstance that the positive direction of $z$ is taken upward.

If, for example, we take a single harmonic component for which $B_{m}=10$ mgals. and $\lambda_{m}=10 \mathrm{~km}$, then $2 \pi B_{m} / \lambda_{m}=63 \times 10^{-9}$. Such a variation in $\Delta g$ as this is frequently encountered and $\Delta(\partial g / \partial z)$ in that case is about 2 per cent of its normal value. The 2 per cent anomaly in the vertical gradient of gravity does never mean an equally 2 per cent change in the resultant gravity anomaly, but it does mean a much greater change. So, if the gravity station is $1000 \mathrm{~m}$ high, for example, the normal free-air reduction to the sea level is 309 mgals., but if $\Delta g$ obtained by that reduction is \pm 10 mgals., the above calculation shows that the free-air reduction is more precisely to be taken as $309 \pm 6$ mgals., i.e. 315 or 303 mgals., according as $\Delta g$ obtained by the first reduction is 10 or -10 mgals. respectively. The more accurate anomalies in gravity will be therefore 16 or -16 mgals. respectively.

The above considerations can easily be extended to the threedimensional cases. For these cases, we get

$$
\begin{gathered}
\Delta U(x y z)=\frac{1}{2 \pi} \sum_{m} \sum_{n} \frac{\lambda_{m} \lambda_{n} B_{m n}}{\sqrt{\lambda^{2}{ }_{m}+\lambda_{n}^{2}} \sin \frac{\cos }{\lambda_{m}} x \sin \lambda_{n} \cos 2 \pi} y \exp \left(-2 \pi \frac{\sqrt{\lambda_{m}^{2}+\lambda_{n}^{2}}}{\lambda_{m} \lambda_{n}} z\right), \\
\Delta g(x y)=\sum_{m} \sum_{n} B_{m n} \cos 2 \pi \lambda_{m} x \sin 2 \pi \\
\sin \lambda_{n}
\end{gathered}
$$

corresponding to (2), (3) and (4) for the two-dimensional cases. According to the above expressions, the two-dimensional distribution of $\Delta(\partial g / \partial z)$ within a certain area can be known as follows: first, expand $\Delta g$ in a double FourIER series, secondly, multiply each of the coefficients in that series by the corresponding $-2 \pi \frac{V \lambda_{m}^{2}+\lambda^{2}}{\lambda_{m} \lambda_{n}}$, and thirdly and lastly, sum up the new series having these new 
coefficients.

In order to see how the method described in the above works for actual problems, it has been applied to the gravity data in U.S.A. The area extending $125^{\circ} \mathrm{W}-77^{\circ} \mathrm{W}$ in longitude and $30^{\circ} \mathrm{N}-$ $48^{\circ} \mathrm{N}$ in latitude covering almost the entire country of U.S.A. may be regarded as a plane rectangle in shape. On the basis of the gravity data published by the U.S. Coast and Geodetic Survey, ${ }^{2)}$ the double FOURIER series coefficients for expressing the distribution of $\Delta g_{0}^{\prime \prime}$ within this extensive area were already calculated. ${ }^{3)}$

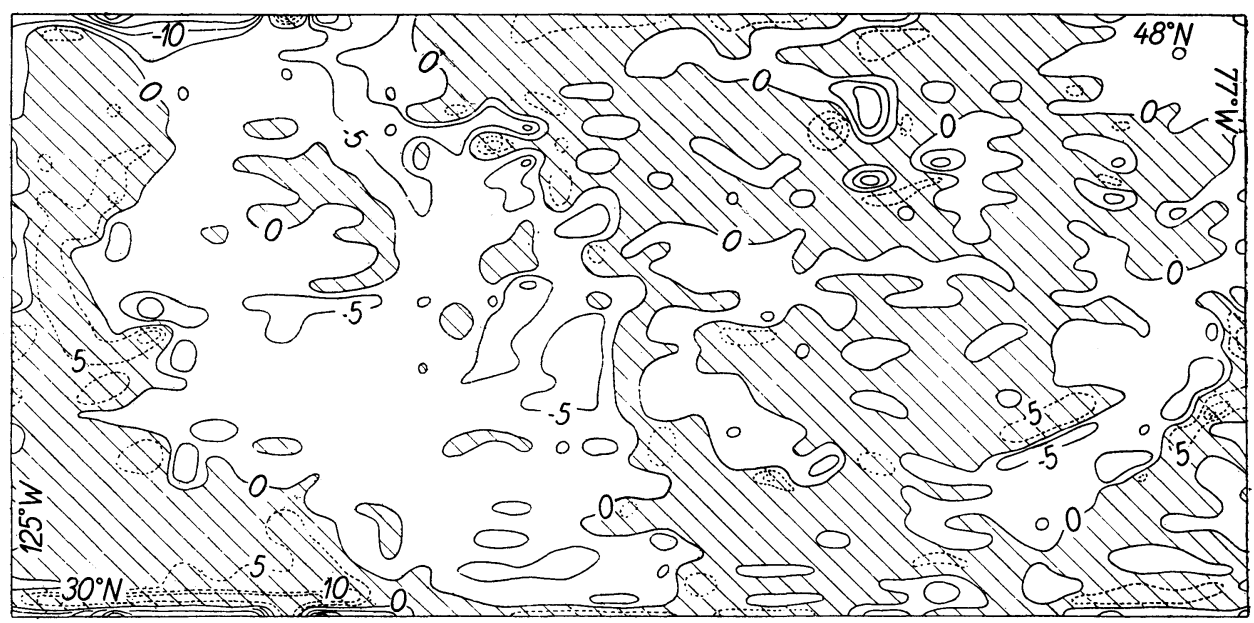

Fig. 1. Calculated distribution of $\Delta\left(\partial g / \partial z\right.$ ) in U.S.A. (in $10^{-9}$ )

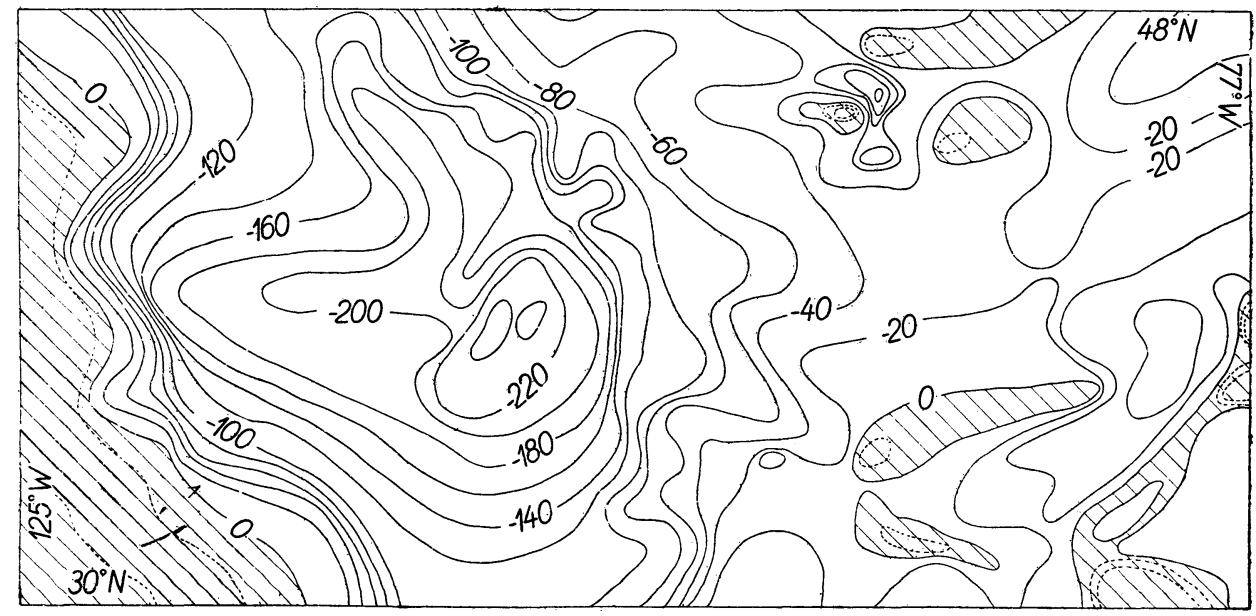

Fig. 2. Observed distribution of $\Delta g_{0}^{\prime \prime}$ in U.S.A. (mgal.)

2) Principal Facts of Gravity Stations in the United States, Parts 1 4 (1938).

3) C. Tsubor et al.: Bull. Earthq. Res. Inst., 17, 385 (1939). 
So, making use of these coefficients and following the method stated in the above, the distribution of $\Delta(\partial g / \partial z)$ in U.S.A. can be determined. At the request of the writer, Mr. T. Kinoshita and Mr. A. HAYATSU have kindly taken the trouble to make the necessary calculations of which the results are shown on the map of Fig. 1 by contour lines. Fig. 2 shows the approximate distribution of $\Delta g_{0}^{\prime \prime}$ in the same area. Since $\Delta(\partial g / \partial z)$ has been calculated from $\Delta g_{0}^{\prime \prime}$ in this case, this may well be called the Bouguer anomaly in the vertical gradient of gravity. The distributions of $\Delta(\partial g / \partial z)$ and of $\Delta g_{0}^{\prime \prime}$ as shown in Figs. 1 and 2 show a close relation with each other as they ought to do.

The largest calculated values of $\Delta(\partial g / \partial z)$ are only about $10 \times 10^{-9}$. It must be noticed, however, that the shortest wave length considered in the calculations by KINoshitA and HAYATSU is as large as $220 \mathrm{~km}$ (18th harmonics) and the effects of those components that have shorter wave lengths have not been taken into account. There do exist much shorter wave length variations in $\Delta g_{0}^{\prime \prime}$ and it is evident that the actual distribution of $\Delta(\partial g / \partial z)$ along the earth's surface is correspondingly much more complicated than the one shown in Fig. 1. Should these short wave length variations be taken into account, the double FOURIER series analysis and synthesis must be extended to include much higher terms and the required numerical calculations will be considerably increased in amount.

Thus, although the above double FourIER series method is useful for studying the approximate distribution of $\Delta(\partial g / \partial z)$ over a wide area, it is not adapted to investigate the local distribution, especially to see what $\Delta(\partial g / \partial z)$ is at a particular station. For this latter purpose, the BESSEL FOURIER series method of analysis which will be developed below will be much competent. By this method, the anomaly in the vertical gradient of gravity at a particular station can be calculated from gravity anomalies around that station.

Let us take the cylindrical co-ordinate with the gravity station in question at its origin. The gravity potential due to a certain anomalous subterranean mass distribution can be expressed in the following BESSEL FOURIER series form:

$$
\Delta U(r \theta z)=\sum_{n}\left\{\int_{0}^{\infty} B_{n k} J_{n}(k r) \exp (-k z) d k\right\}_{\sin }^{\cos } n \theta,
$$

using customary notations. From this expression for the potential, we get at the earth's surface $z=0$,

$$
\Delta g(r \theta)=\sum_{n}\left\{\int_{0}^{\infty} B_{n k} J_{n}(k r) d k\right\} \begin{aligned}
& \cos \\
& \sin
\end{aligned} \theta
$$




$$
\begin{aligned}
=\int_{0}^{\infty} B_{0 k} J_{0}(k r) d k+ & \left\{\int_{0}^{\infty} B_{1 k} J_{1}(k r) d k\right\}_{\sin }^{\cos } \theta \\
& +\left\{\int_{0}^{\infty} B_{2 k} J_{2}(k r) d k \int_{\sin }^{\cos } 2 \theta+\cdots,\right.
\end{aligned}
$$

and

$$
\begin{aligned}
\Delta(\partial g / \partial z)(r \theta) & =-\sum_{n}\left\{\int_{0}^{\infty} k B_{n k} J_{n}(k r) d k\right\}_{\sin }^{\cos } n \theta \\
= & -\int_{0}^{\infty} k B_{0 k} J_{0}(k r) d k-\left\{\int_{0}^{\infty} k B_{1 k} J_{1}(k r) d k\right\}_{\sin }^{\cos } \theta \\
& -\left\{\int_{0}^{\infty} k B_{2 k} J_{2}(k r) d k\right\}_{\sin }^{\cos 2 \theta \ldots .}
\end{aligned}
$$

In order to see what the values at the origin will become, we make $r$ in (10) tend to zero. In doing this, we make use of the properties of the BESSEL functions such as

$$
\begin{aligned}
& J_{0}(0)=1, \\
& J_{1}(0)=J_{2}(0)=\cdots=0 .
\end{aligned}
$$

Then, at $r=0$, all the terms on the right hand side vanish except the first, and (10) becomes simply

$$
\Delta(\partial g / \partial z)_{0}=-\int_{0}^{\infty} k B_{0 k} d k
$$

A little further mathematics shows that (11) can be written in the form:

$$
\Delta(\partial g / \partial z)_{0}=-\int_{0}^{\infty} \frac{d r}{r^{2}} \int_{0}^{\infty} B_{0 k}\left\{1-J_{0}(k r)\right\} d k .
$$

Now, if (9) is integrated with respect to $\theta$ from 0 to $2 \pi$ and divided by $2 \pi$, we get

$$
2 \pi \int_{0}^{2 \pi} \Delta g(r \vartheta) d \theta=\int_{0}^{\infty} B_{0 k} J_{0}(k r) d k .
$$

This is the average of $\Delta g(r \theta)$ taken over the whole azimuth $\theta$ along a circle $r=r$, drawn around the origin. Let this average be denoted by $\overline{\Delta g(r)}$. Since

$$
\overline{\Delta g(0)}=\int_{0}^{\infty} B_{0 k} d k
$$

4) To prove this, the relation

is made use of.

$$
\int_{0}^{\infty} \frac{\left\{1-J_{0}(k r)\right\}}{r^{2}} d r=k
$$


and

$$
\overline{\Delta g(r)}=\int_{0}^{\infty} B_{0 k} J_{0}(k r) d k
$$

from (13), we see

$$
\overline{\Delta g(0)}-\overline{\Delta g(r)}=\int_{0}^{\infty} B_{0 k}\left\{1-J_{0}(k r)\right\} d k .
$$

Putting (16) into (12), we get

$$
\Delta(\partial g / \partial z)_{0}=-\int_{0}^{\infty}\{\overline{\Delta g(0)}-\overline{\Delta g(r)}\} \frac{d r}{r^{2}} .
$$

But, owing to the property of $J_{0}(k r),\{\overline{\Delta g(0)}-\overline{\Delta g(r)}\}$ varies as $\alpha r^{2}$ in the neighbourhood of $r=0$, say up to $r=R$. Thus, the above integration in (17) may be split into two parts and may be written as

$$
\begin{aligned}
\Delta(\partial g / \partial z)_{0} & =-\left[\int_{0}^{R} \frac{\alpha r^{2}}{r^{2}} d r+\int_{R}^{\infty}\{\Delta g(0)-\overline{\Delta g(r)}\} \frac{d r}{r^{2}}\right] \\
& =-\left[\frac{\{\overline{\Delta g(0)}-\overline{\Delta g(R)\}}}{R}+\int_{K^{2}}^{\infty} \frac{\{\overline{\Delta g(0)}-\overline{\Delta g(r)}\}}{r^{2}} d r\right] .
\end{aligned}
$$

This is the expression useful for an accurate evaluation of $\Delta(\partial g / \partial z)_{0}$ from $\Delta g$. An essentially same expression as this was already obtained by H. M. EvJEN. ${ }^{5)}$ But he did not consider the effect of $\overline{\Delta g(0)}$ and wrote simply

$$
\Delta(\partial g / \partial z)_{0}=\int_{0}^{\infty} \frac{\Delta g(r)}{r^{2}} d r .
$$

Also EvJEN took $R$ in such a way that up to that $R, \overline{\Delta g(r)}$ is approximately constant. From our considerations, $R$ can be extended to such a limit up to which $\left\{\overline{\Delta g(0)}-\overline{\Delta g(r)\}}\right.$ varies as $\alpha r^{2}$.

Now let us investigate how the present method works in actual problems. In order to do this, a good map showing the detailed distribution of $\Delta g$ within a possibly large area is needed. It has been found that the map which was published by Donald A. RICE ${ }^{6)}$ is one that satisfies our requirements. RICE's map covers an area extending more than $10^{6} \mathrm{~km}^{2}$ in the south-western part of U.S.A. and the details of the distribution of the free-air anomalies of gravity are shown on it by contour lines, based on a great many gravity measurements, for this is probably one of the areas which have been most extensively explored in the world by the gravimetric methods.

5) H. M. Evjen: Geophysics, 1, 127 (1936).

6) Donald A. RICE: Deflections of the Vertical from Gravity Anomalies (1951). —: Bull. géod., 25, 285 (1952). 
The point at $\varphi=34^{\circ} .3 \mathrm{~N}$ and $\lambda=98^{\circ} .3 \mathrm{~W}$, (at the centre of the closed contour line of 70 mgals.) on RICE's map has been selected to be the test point for our calculations. This selection has been made because the point is situated at about the middle of the map so that $\Delta g$ up to reasonably large distances from that may be taken into account and also because $\Delta g$ is a maximum (about 70 mgals.) at this point so that a fairly large value of $\Delta(\partial g / \partial z)$ is expected.

From the map of RICE, the values of $\Delta g$ at 24 points along each of the concentric circles drawn at every $20 \mathrm{~km}$ distance interval around the point have been read off and $\overline{\Delta g(r)}$ calculated. The values of $\{\overline{\Delta g(0)}-\overline{\Delta g(r)}\}$, as well as of $\frac{1}{r^{2}}\{\overline{\Delta g(0)}-\overline{\Delta g(r)}\}$ and $\int_{R}^{r}\{\Delta g(0)-\Delta g(r)\} \frac{d r}{r^{2}}$ are plotted in Fig. 3 against $r$.

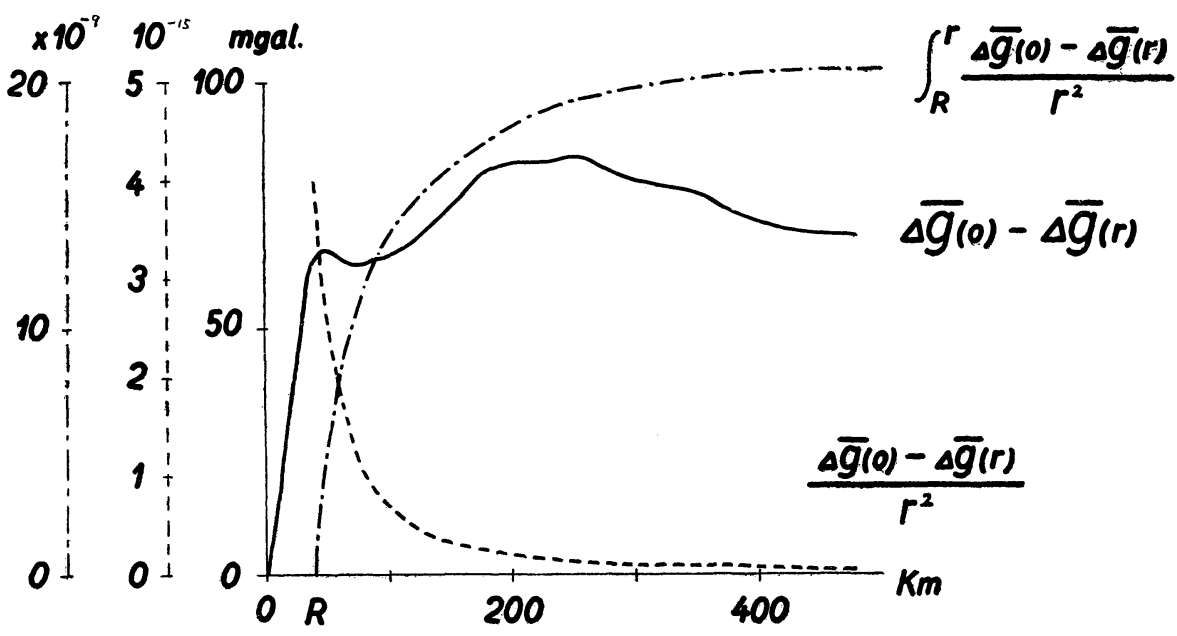

Fig. 3

Looking at Fig. 3, we shall regard $\{\overline{\Delta g(0)}-\overline{\Delta g(r)\}}$ as varying in a parabolic manner up to $R=40 \mathrm{~km}$ and we see that $\{\overline{\Delta g(0)}-\overline{\Delta g(R)}\}$ is 63 mgals. The first term in the bracket of (18) is therefore

$$
\frac{63 \times 10^{-3}}{40 \times 10^{5}}=16 \times 10^{-9} \text {. }
$$

The integral of the second term in the bracket has been evaluated numerically and found to be $21 \times 10^{-9}$. Thus finally we get

$$
\Delta(\partial g / \partial z)_{0}=-(16+21) \times 10^{-9}=-37 \times 10^{-9} .
$$

At this particular station in question $\Delta(\partial g / \partial z)$ has thus been found to be a little more than one per cent of the normal value. Even 
the anomaly of this magnitude will result in the difference of 3 mgals. in the free-air reduction of gravity value to the sea level, if the station is $1000 \mathrm{~m}$ high. The difference of this amount can never be overlooked, if we wish to obtain accurate knowledges regarding the distribution of gravity anomalies. In conclusion, it may be added that since $\Delta(\partial g / \partial z)$ has been calculated from $\Delta g_{0}$, this may well be called the free-air anomaly of the vertical gradient of gravity.

The writer thanks Mr. T. Kinoshita and Mr. A. Hayatsu, post-graduate students in this laboratory, who have helped the writer in preparing this article. 